\title{
Anorexia nervosa presenting as foot drop
}

\author{
G. D. SCHOTT \\ M.D., M.R.C.P. \\ Department of Neurology, King's College Hospital, London
}

\begin{abstract}
Summary
A patient with anorexia nervosa is reported whose presentation with foot drop was to a neurological department. Bilateral peroneal nerve palsies were subsequently demonstrated. The aetiology of these transient palsies is discussed.
\end{abstract}

\section{Introduction}

Anorexia nervosa is undoubtedly a commoner condition than is often realized. Although the condition usually becomes manifest through its psychiatric features, it may declare itself in a number of unusual ways. The patient described below is an example of this.

\section{Case report}

A 15-year-old girl presented to the neurological department with a 3-month history of difficulty in walking owing to foot drop affecting the right leg. She had a tendency to trip and catch the toes of the right foot and a few weeks after the onset had been troubled by paraesthesiae affecting the lateral aspect of the right lower leg. There had been no history of trauma or unusual activities involving the legs before the fairly sudden onset of her symptoms. Further direct questioning, however, revealed a history of secondary amenorrhoea of 4 months' duration, and for the same period she had been irritable and depressed, with marked lethargy, passivity and cold intolerance and had noticed some loss of hair from the head. She had become giddy on standing and had been constipated for one month. Formal psychiatric assessment elicited that the patient, who had eaten heartily until puberty, had for 2 years made attempts at dieting which were usually given up. She had developed a fear of becoming fat and described very ambivalent feelings in growing up, finding friends and forming relationships. She was clinging to her parents and lacked the normal adolescent drive for independence.

On examination she looked severely ill, emaciated and miserable. Her weight was $39 \mathrm{~kg}$, having some months previously been about $50 \mathrm{~kg}$. She had lost

Correspondence: Dr G. D. Schott, The National Hospital for Nervous Diseases, Queen Square, London WC1N 3BG. normal female body shape and was strikingly thin, $\overrightarrow{\vec{H}}$ with marked loss of subcutaneous tissue particularly from the face. Her rectal temperature was $33.5^{\circ} \mathrm{C}$, 항 her pulse rate 41 beats/min and her lying and stand- 3 ing blood pressure $80 / 50 \mathrm{mmHg}$. There was normal if axillary and pubic hair but head hair was sparse $\sigma$ and fine hair was evident over the lower legs. She $\underset{\mathcal{~}}{ }$ had a dry skin. Neurological examination revealed of complete foot drop with paralysis of the foot dorsiflexors and evertors on the right; in the left leg, of $\frac{}{2}$ which the patient had not complained, weakness of $\vec{c}$ ankle dorsiflexion (MRC Grade 2) and eversion (Grade 3) was also evident. There was impairment of cutaneous sensation over the antero-lateral aspect $₹$ of the right calf. The common peroneal nerves were $\overrightarrow{0}$ easily palpable although not thickened. Tendon re flexes were present but sluggish.

Investigations revealed a normal blood picture ESR, plasma electrolytes, urea, SMA 12 metabolic screen and lipid profile. Radiographs of chest, skull, pituitary fossa and hands and an isotope brain scan $\frac{\mathscr{}}{\varnothing}$ were normal. Blood serological tests for syphilis $\varrho$ and a urine pregnancy test were negative. ECG con- $\overrightarrow{\overrightarrow{0}}$ firmed sinus bradycardia of 41 beats $/ \mathrm{min}$ with a 3 normal QTc interval. Endocrine studies revealed normal serum thyroxin, prolactin, midnight and 9 a.m. plasma cortisols and 24-hr urinary free $\frac{\partial}{2}$ cortisol. Growth hormone concentration $(9 \mathrm{mu} . / \mathrm{l}) \stackrel{3}{-}$. was at the upper limit of normal. TSH was less than $0.5 \mathrm{mu} . / 1$. Whilst the basal FSH level was normal, the LH level was slightly reduced $(1.7 \mathrm{u} . / \mathrm{l})$ although $\frac{\text { o }}{3}$ both hormone levels rose normally with $100 \mu \mathrm{g}$ 웅 releasing hormone.

Electrophysiological studies on admission demon- $\frac{D}{0}$ strated bilateral common peroneal nerve damage at the fibular head but no evidence of a generalized $N$ peripheral neuropathy. The distal motor latency to the right tibialis anterior was $15.7 \mathrm{msec}(10 \mathrm{~cm}) \stackrel{\mathrm{N}}{\mathrm{N}}$ and the amplitude of the evoked response $0.4 \mathrm{mV}$. $\omega$ Values for the left leg were $4.6 \mathrm{msec}$ and $2 \mathrm{mV}$ ? respectively. The left sural nerve sensory action $\stackrel{0}{=}$ potential had an amplitude of $16 \mu \mathrm{V}$ with a maxi- $\stackrel{\Phi}{\infty}$ mum conduction velocity of $33 \mathrm{~m} / \mathrm{sec}$, the skin temperature at the recording site being $27^{\circ} \mathrm{C}$. There was evidence of severe denervation of both tibialis $\underset{\mathbb{D}}{\stackrel{\mathrm{D}}{0}}$ 
anterior muscles but not of the gastrocnemii. Further studies 3 weeks later when the patient was normothermic showed normal amplitude, form and latency of the median and sural sensory action potentials and normal median sensory conduction velocity $(54 \mathrm{~m} / \mathrm{sec})$. A study of the left common peroneal nerve at this second investigation showed a pronounced decrement of the evoked response picked up over the extensor digitorum brevis on stimulation proximal to the fibular head, the responses being $3.4 \mathrm{mV}$ and $1.4 \mathrm{mV}$ on stimulation at ankle and popliteal fossa respectively; distal motor latency and motor conduction velocity were normal (41 $\mathrm{m} / \mathrm{sec}$ ).

Psychological assessment revealed a verbal i.q. of 85 and performance i.q. of 96 on the Wechsler Intelligence Scale for Children. She did poorly on the subtests requiring good educational skills and her reading age was 11.5 years on the Schonell Diagnostic Reading Test. Her attitude towards body shapes was found to be normal, and she recognized herself as underweight; her conception of the average shape was normal.

Although the patient and her parents initially denied abnormal eating habits, weight loss or psychiatric factors, a diagnosis of anorexia nervosa appeared inescapable. No evidence for an underlying systemic illness was forthcoming and a superficial resemblance to hypopituitarism was excluded both clinically and by investigation. In-patient management was at first directed to slow warming and encouragement to eat. Her hypothermia disappeared over $48 \mathrm{hr}$, but for one month her pulse rate tended to fluctuate between 50 and 90 beats $/ \mathrm{min}$ and her blood pressure remained about $90 / 50 \mathrm{mmHg}$. During this period her foot drop improved satisfactorily and the sensory features disappeared, although examination continued to show moderate weakness of the affected muscles. Five months after presentation there remained only barely detectable weakness of foot dorsiflexion (Grade 4+) affecting the right leg more than the left. Electrophysiological studies provided evidence of active nerve terminal regeneration, although a considerable degree of conduction block at the fibular head was still present, especially on the right. Although her neurological condition had markedly improved, her weight increase was less satisfactory. Thus she was discharged from the ward after one month having refused further in-patient treatment, and gained only $3 \mathrm{~kg}$ over the subsequent 6 months notwithstanding weekly outpatient psychiatric care.

\section{Discussion}

Despite the absence of presenting psychiatric symptoms, there can be little doubt that clinically this patient suffered from anorexia nervosa (Dally,
1967; Leading Article, 1975). Although, as in this case, there may be some superficial resemblance to hypopituitarism, several subtle presentations that may conceal this diagnosis have been described, including drug dependency, epilepsy, unexplained diarrhoea or vomiting, periodic oedema, polydipsia, amenorrhoea, ritualistic behaviour and depression (Crisp, Palmer and Kalucy, 1976). The present patient appears to have presented with a hitherto undescribed complication of anorexia nervosa. The peroneal nerve palsies, one of which was asymptomatic, can be attributed to nerve compression at the fibular head, presumably from crossing the legs or similar factors causing local pressure.

It is perhaps surprising that foot drop is not a more common manifestation of anorexia nervosa, considering the increased vulnerability of the more exposed nerves consequent upon the marked subcutaneous tissue loss that occurs. It is of interest that emaciated prisoners-of-war (in the absence of vitamin deficiency) have been found to develop peroneal nerve palsies when spending long periods of time squatting or sitting with their legs crossed but not when remaining active (Kaminsky, 1947). The fairly prolonged period of inertia and relative immobility in the present patient may thus have contributed to the development of her symptoms. Peroneal nerve palsy is also a recognized hazard of weight reduction, particularly when therapeutic weight-reducing diets and prolonged inactivity are combined (Sprofkin, 1958). This inactivity has been stressed as an important factor, and it is noteworthy that peroneal nerve palsy has been observed in association with weight loss occurring as part of a depressive illness (Woltman, 1929). Fortunately the prognosis for recovery is usually good, and it will be recalled that in this patient recovery occurred despite maintenance of a low body weight. Presumably partial refeeding, resumption of activity and avoidance of further local trauma to the nerves contributed to the resolution of her symptoms.

Anorexia nervosa has attracted considerable interest predominantly in psychiatric practice for many decades, although the precise aetiology remains elusive. The patient reported here illustrates an unusual presentation of this enigmatic condition.

\section{Acknowledgments}

I am indebted to Professor C. D. Marsden and Dr L. Wheelan for permission to publish details of this patient. It is a pleasure to thank Dr Wheelan for advice on the psychiatric aspects, Dr C. G. McKerron for help with the endocrinological assessment, and Dr J. Payan for kindly performing the electrophysiological investigations.

\section{References}

Crisp, A.H., Palmer, R.L. \& Kalucy, R.S. (1976) How common is anorexia nervosa? A prevalence study. British Journal of Psychiatry, 128, 549. 
Dally, P.J. (1967) Anorexia nervosa-long-term follow-up and effects of treatment. Journal of Psychosomatic Research, 11, 151.

Kaminsky, F. (1947) Peroneal palsy by crossing the legs. Journal of the American Medical Association, 134, 206.

LeADING Article (1975) Hormone patterns in anorexia nervosa. British Medical Journal, 2, 52.

SPRofkin, B.E. (1958) Peroneal paralysis - a hazard of weight reduction. Archives of Internal Medicine, 102, 82.

Woltman, H.W. (1929) Crossing legs as factor in production of peroneal palsy. Journal of the American Medical $\subseteq$ Association, 93, 670. 